\title{
Proteomic comparison of Mycobacterium avium subspecies paratuberculosis grown in vitro and isolated from clinical cases of ovine paratuberculosis \\ Correspondence \\ Valerie Hughes \\ Val.Hughes@moredun.ac.uk \\ Received 10 May 2006 \\ Revised 16 August 2006 \\ Accepted 21 August 2006

\author{
Valerie Hughes, ${ }^{1}$ Stuart Smith, ${ }^{1}$ Alfredo Garcia-Sanchez, ${ }^{1} \dagger$ Jill Sales ${ }^{2}$ \\ and Karen Stevenson ${ }^{1}$
${ }^{1}$ Moredun Research Institute, Pentlands Science Park, Bush Loan, Penicuik EH26 OPZ, UK
${ }^{2}$ Biomathematics and Statistics Scotland, James Clerk Maxwell Building, The King's Buildings, Edinburgh EH9 3JZ, UK

\begin{abstract}
Paratuberculosis (Johne's disease) poses a significant economic problem to beef, dairy and sheep industries worldwide, and is caused by Mycobacterium avium subspecies paratuberculosis. In this study, 2D PAGE was used as a tool to investigate the virulent state of $M$. avium subsp.

paratuberculosis, incorporating the technique of beating the organism with zirconium/silica beads to provide a comprehensive representation of its proteome. A direct comparison of the proteomes of $M$. avium subsp. paratuberculosis scraped from the terminal ileum of ovine paratuberculosis cases, and the identical strain grown in vitro, is presented. These analyses identified a set of 10 proteins whose expression is upregulated during natural infection: 1-pyrroline-5-carboxylate dratase (2-mcd), arginosuccinate synthase (ArgG), universal stress protein (usp), 30S ribosomal same pathway, and may have a concerted action for energy production in vivo.
\end{abstract} dehydrogenase (RocA), a putative acyl-CoA dehydrogenase (FadE14), 2-methylcitrate dehy- protein S2 (RpsB), peptidyl-prolyl cis-trans isomerase (PpiA), luciferase-like monooxygenase (Imo), thiosulfate sulfurtransferase (SseA) and ATP-dependent Clp protease (ClpB). Most of the proteins identified do not have obviously related functions; however, ArgG and RocA function in the
}

\section{INTRODUCTION}

Paratuberculosis (Johne's disease) poses a significant economic problem to beef, dairy and sheep industries worldwide. The clinical symptoms are chronic enteritis (with persistent diarrhoea in cattle), reduced feed intake, and severe weight loss. The disease is caused by Mycobacterium avium subspecies paratuberculosis and has a complex aetiology. Infected animals may be asymptomatic, and only $3-10 \%$ of infected animals exhibit clinical symptoms (Julian, 1975; Chiodini et al., 1984). In addition to this, clinical ovine paratuberculosis exhibits two forms: the multibacillary form in which many acid-fast organisms, as determined by Ziehl-Neelsen (ZN) staining, are present;

tPresent address: Patología Infecciosa, Facultad de Veterinaria Universidad de Extremadura, Avda de la Universidad s/n, 10071 Cáceres, Spain

Abbreviations: 2-mcd, 2-methylcitrate dehydratase; CBB, Coomassie brilliant blue; Imo, luciferase-like monooxygenase; NCBI, National Center for Biotechnology Information; usp, universal stress protein; ZN, Ziehl-Neelsen.

Spot volumes of all gels and an additional gel image are available as supplementary data with the online version of the paper. and the paucibacillary form in which few acid-fast organisms are present in the terminal ileum, the primary site of pathogenesis (Clarke, 1997)

Infectious disease is the consequence of a finely tuned and orchestrated interplay between host and pathogen. Adaptation of the organism to the environment provided by the host, and the temporal, tissue-specific interplay between pathogen virulence and host defences, will determine the outcome of infection, whether it be clinical disease or asymptomatic carriage. Understanding this interaction at the molecular level will identify mechanisms of pathogenesis that promote bacterial survival in host tissue. The molecules identified may then become targets for intervention in the control of infectious disease.

Global strategies have the potential to identify genes with functions as yet unrevealed in host-bacteria interactions. In vivo expression technology is the only established method to identify genes differentially expressed in bacteria within infected animals. This powerful technology has increased our understanding of the pathogenic processes of a number of organisms, and has identified proteins that promote bacterial survival in host tissue (Angelichio \& Camilli, 
2002). However, in vivo expression technology requires genetic manipulation of the pathogen before infection, by homologous recombination or stable maintenance of plasmids to enable promoter-reporter fusions to be selected within the host environment. M. avium subsp. paratuberculosis has so far proven very difficult to transform and genetically modify. Furthermore, establishing an experimental infection with $M$. avium subsp. paratuberculosis is problematic. Thus, another more direct approach is necessary to identify proteins the levels of which are modified in vivo.

We used 2D PAGE as a tool to investigate the virulent state of $M$. avium subsp. paratuberculosis, incorporating the technique of beating the organism with zirconium/silica beads to provide a comprehensive representation of its proteome. Here, we present a direct comparison of the proteomes of M. avium subsp. paratuberculosis scraped from the terminal ilea of clinical cases of ovine paratuberculosis with those from the organism isolated from the same animals and grown in standard laboratory culture.

\section{METHODS}

Diagnosis and storage of intestines. Five animals with suspected disease were obtained from flocks known to be infected with M. avium subsp. paratuberculosis. Confirmation of paratuberculosis in these animals was made following culture, PCR and histopathological examination of tissues obtained post-mortem. Animals were euthanized with an intravenous injection of $100 \mathrm{mg}$ pentobarbital $\mathrm{kg}^{-1}$. Approximately $30 \mathrm{~cm}$ of terminal ileum was removed postmortem and flushed with water to remove contaminating debris. Small sections of the tissue were removed for bacteriological and histopathological examination to confirm the diagnosis, and to determine whether the multibacillary or paucibacillary form was present. For histopathology, tissues were fixed in $10 \%(\mathrm{v} / \mathrm{v})$ formol saline for a minimum of $24 \mathrm{~h}$, trimmed, dehydrated through graded alcohols, and embedded in paraffin wax. Sections $5 \mu \mathrm{m}$ thick were cut and stained with haematoxylin and eosin. Serial sections were stained by the $\mathrm{ZN}$ method. The intestines were stored frozen at $-20{ }^{\circ} \mathrm{C}$ until bacteriology and histopathology reports were available. Only the multibacillary form, characterized by the presence of a mycobacteria-loaded macrophage infiltrate with few $\mathrm{T}$ cells, was used in this study.

Isolation of M. avium subsp. paratuberculosis strains from terminal ileum of clinical paratuberculosis cases. Fat and connective tissue were removed from $1 \mathrm{~cm}^{2}$ sections of terminal ileum from animals diagnosed with clinical paratuberculosis. The tissue was roughly chopped and homogenized in $10 \mathrm{ml}$ distilled water using a Colworth Stomacher 80 at normal settings for $30-60 \mathrm{~s}$. The homogenate was treated with an equal volume of $1.5 \%(\mathrm{w} / \mathrm{v})$ hexadecylpyridinium chloride and allowed to settle and decontaminate overnight. The decontaminated suspension was removed to a fresh tube, avoiding any settled material, and then centrifuged $(3000 \mathrm{~g}, 20 \mathrm{~min}$, room temperature). The resultant pellet was washed in distilled water twice with recentrifugation, and finally resuspended in $0.5 \mathrm{ml}$ distilled water. The suspension $(0.1 \mathrm{ml})$ was then used to inoculate duplicate slopes of Middlebrook 7H11 agar supplemented with $20 \%(\mathrm{v} / \mathrm{v})$ newborn calf serum (Invitrogen), heat-inactivated at $56^{\circ} \mathrm{C}$ for $1 \mathrm{~h}, 10 \%(\mathrm{v} / \mathrm{v})$ Middlebrook oleic acid/ albumin/dextrose/catalase enrichment medium (Difco), $2.5 \%(\mathrm{v} / \mathrm{v})$ glycerol, $0.03 \%(\mathrm{w} / \mathrm{v})$ asparagine, $2 \mu \mathrm{g}$ Mycobactin $\mathrm{J} \mathrm{ml}^{-1}$ (Allied
Monitor) and two Selectatabs (MAST Laboratories) per litre. Cultures were incubated at $37^{\circ} \mathrm{C}$ until colonies were visible (up to 16 weeks). In order to check that the isolates were M. avium subsp. paratuberculosis, a diagnostic PCR-based test for the detection of the specific insertion sequence IS900 was performed. All samples were found to be positive for IS900.

In vitro growth of $\boldsymbol{M}$. avium subsp. paratuberculosis. Starter cultures were initiated by inoculating a loopful of mycobacteria (from a 7H11 slope with confluent growth) into a $10 \mathrm{ml}$ volume of Middlebrook 7H9 medium supplemented with $10 \% \quad(\mathrm{v} / \mathrm{v})$ Middlebrook oleic acid albumin/dextrose/catalase enrichment medium, $0.1 \%(\mathrm{w} / \mathrm{v})$ Tween-80, $2 \mu \mathrm{g}$ Mycobactin $\mathrm{J} \mathrm{ml}^{-1}$ and $2.5 \%$ $(\mathrm{v} / \mathrm{v})$ glycerol. The cultures, contained in $50 \mathrm{ml}$ flasks, were stirred continuously using magnetic stirrer bars, on a multiple-position, low-heat-transmission magnetic stirrer. After $48 \mathrm{~h}$ incubation, $3 \mathrm{ml}$ starter culture was transferred to $300 \mathrm{ml}$ pre-warmed medium in 11 flasks. The cultures were incubated at $37^{\circ} \mathrm{C}$ with continuous stirring until they reached exponential or stationary growth phase. The conditions described above provided optimal in vitro growth conditions.

Determination of growth phase by OD. $\mathrm{OD}_{600}$ was measured at appropriate time points throughout the growth of the $300 \mathrm{ml}$ liquid cultures. Small aliquots of the culture $(0.2 \mathrm{ml})$ were removed aseptically, diluted with $0.1 \%(\mathrm{w} / \mathrm{v})$ Tween- $80(0.8 \mathrm{ml})$ and the $\mathrm{OD}_{600}$ ascertained ( $1 \mathrm{~cm}$ path length cuvette, Novaspec II; GE Healthcare). The $\mathrm{OD}_{600}$ of liquid cultures of $M$. avium subsp. paratuberculosis was plotted against time, and a typical growth curve was generated. This extremely slow-growing organism exhibits the three standard phases of growth: the initial lag phase with little change in $\mathrm{OD}_{600}$ with respect to time; the exponential phase, in which the $\mathrm{OD}_{600}$ increases exponentially with respect to time, generally peaking at $\mathrm{OD}_{600}$ 2.5-3; and the stationary phase, in which the growth rate slows and the OD of the culture declines. The majority of M. avium subsp. paratuberculosis strains exhibit similar rates of growth; however, the lag phase can be very protracted for certain strains.

Harvesting in vitro-grown M. avium subsp. paratuberculosis for proteomic analysis. When cultures of $M$. avium subsp. paratuberculosis were determined to have reached either exponential or stationary phase, and to have achieved $O D \geqslant 1$, they were harvested. Aliquots $(50 \mathrm{ml})$ were removed aseptically to $50 \mathrm{ml}$ conical tubes. These were cooled on ice, and then centrifuged $(4000 \mathrm{~g}, 30 \mathrm{~min}$, $4{ }^{\circ} \mathrm{C}$ ). The supernatant was decanted, and the cell pellet was carefully resuspended in $25 \mathrm{ml}$ ice-cold PBS (8 g sodium chloride $\mathrm{l}^{-1}, 0.2 \mathrm{~g}$ potassium chloride $1^{-1}, 1.15 \mathrm{~g}$ disodium hydrogen phosphate $1^{-1}$, $0.2 \mathrm{~g}$ potassium dihydrogen phosphate $\left.1^{-1}\right)$. The cell suspension was then centrifuged again $\left(4000 \mathrm{~g}, 30 \mathrm{~min}, 4^{\circ} \mathrm{C}\right)$, the supernatant removed, and the pellet stored at $-20^{\circ} \mathrm{C}$. Cell pellets were stored for no longer than 1 week before further processing for proteomic analysis.

Purification of $M$. avium subsp. paratuberculosis from the terminal ileum of clinical cases of ovine paratuberculosis. This was performed essentially as described by Choy et al. (1998). Briefly, frozen gut was rapidly thawed and retained on ice during processing. Sections of $4-5 \mathrm{~cm}$ length were cut from the gut and were opened opposite the mesenteric joint face. The mucosal gut was scraped with a microscope slide, and the scrapings were homogenized in $0.2 \mathrm{M}$ sucrose ( $\sim 5 \mathrm{~g}$ mucosal scraping per $20 \mathrm{ml}$ sucrose solution).

The homogenate was centrifuged $\left(200 \mathrm{~g}, 20 \mathrm{~min}, 4{ }^{\circ} \mathrm{C}\right)$. The pellet was mixed on a vortex mixer with a further $20 \mathrm{ml} 0.2 \mathrm{M}$ sucrose and was recentrifuged. Both supernatants were combined, mixed thoroughly, and then centrifuged $\left(5500 \mathrm{~g}, 10 \mathrm{~min}, 4^{\circ} \mathrm{C}\right)$ over a step gradient of $0.3 \mathrm{M}$ sucrose. The pellets were resuspended in $0.2 \mathrm{M}$ sucrose, and 
centrifuged $\left(5500 \mathrm{~g}, 10 \mathrm{~min}, 4^{\circ} \mathrm{C}\right)$ twice through a step gradient of $1.5 \mathrm{M} \mathrm{KCl}$, washed twice in PBS, and then twice with sterile distilled water. Purity of the preparation was assessed by ZN staining and light microscopy. Samples were either processed directly or stored at $-20{ }^{\circ} \mathrm{C}$ until required.

Protein extraction. Cells $(0.2-0.3$ g wet weight $)$ were suspended in $2 \%$ (w/v) SDS, $0.04 \mathrm{M}$ Tris, $0.06 \mathrm{M}$ DTT. The suspension was layered onto washed $0.1 \mathrm{~mm}$ zirconium/silica beads (Biospec Products). The mixture was lysed six times using a Fastprep 120 (Qbiogene) at 6.5 beats per second, with 1 min cooling on ice between each round. The resulting suspension was centrifuged $(500 \mathrm{~g}, 30 \mathrm{~s}$, room temperature) to reduce frothing. The supernatant was pipetted into microcentrifuge tubes and heated to $100{ }^{\circ} \mathrm{C}$ for $5 \mathrm{~min}$. It was then rapidly cooled and centrifuged $(21000 \mathrm{~g}, 20 \mathrm{~min}$, room temperature). The protein extract was stored at $-70{ }^{\circ} \mathrm{C}$ until required.

Protein clean-up. This procedure was performed as described in the protocol accompanying the PlusOne 2-D Clean-Up kit (GE Healthcare).

Protein solubilization. The pellet obtained after the clean-up procedure was dissolved in $8 \mathrm{M}$ urea and $4 \%$ (w/v) CHAPS, which contained one tablet of Complete, Mini Protease Inhibitor Cocktail (Roche) per $10 \mathrm{ml}$ solubilization buffer. The protein suspension was incubated at $25^{\circ} \mathrm{C}$ for $60 \mathrm{~min}$. Following solubilization, the suspension was centrifuged briefly $(10000 \mathrm{~g}, 1 \mathrm{~min}$, room temperature) to pellet the residual insoluble material. Soluble proteins were then reduced by incubation with tris-(2-carboxyethyl)phosphine hydrochloride for $30 \mathrm{~min}$, added to a final concentration of $0.002 \mathrm{M}$, followed by alkylation with iodoacetamide, which was added to a final concentration of $0.015 \mathrm{M}$, and incubated for $45 \mathrm{~min}$ at room temperature. After incubation, the solution was centrifuged $(21000 \mathrm{~g}$, $20 \mathrm{~min}, 16^{\circ} \mathrm{C}$ ) to remove any residual particulate material. Soluble protein extracts were used immediately or stored at $-70{ }^{\circ} \mathrm{C}$.

Protein determination. The concentration of protein extracts was determined using the PlusOne Quant kit (GE Healthcare), and was performed essentially as described in the protocol accompanying the product. The results of the assay were routinely confirmed by visual assessment of the protein loaded onto a 1D SDS-PAGE gel. This was performed because occasionally the assay overestimated the amount of protein present in mycobacterial preparations from the ileum of animals, probably due to impurities carried over in the clean-up procedure.

Optimization of the protein extraction procedure. Protein extraction was initially performed in the solution used for protein solubilization; however, the quality of the resulting $2 \mathrm{D}$ gel profiles was reduced by horizontal streaking of proteins with an isoelectric point (pI) $<6$ and a molecular mass $>30 \mathrm{kDa}$ (data not shown). This was consistent with the presence of contaminating nonproteinaceous impurities, and can be seen elsewhere in M. avium subsp. paratuberculosis 2D protein profiles (Lanigan et al., 2004). By employing the extraction and clean-up procedures described above, we succeeded in reducing the horizontal streaking to a minimum, enabling subsequent gel comparison.

Optimization of 2D PAGE. After preliminary experiments with IEF strips of pI 3-10 and 3-10 'non linear', we found that the best and most reproducible separation of spots in the second dimension was obtained after performing the IEF on $24 \mathrm{~cm}$ strips of pI $4-7$.

IEF. Protein [150-200 $\mu \mathrm{g}$ for silver-stained gels, or 300-500 $\mu \mathrm{g}$ for colloidal Coomassie brilliant blue (CBB)-stained gels] was incubated in $0.45 \mathrm{ml} 1 \%(\mathrm{v} / \mathrm{v})$ immobilized $\mathrm{pH}$ gradient buffer, $\mathrm{pH} 4-7$ (GE Healthcare), $0.037 \mathrm{M}$ DTT, $4 \%(\mathrm{w} / \mathrm{v})$ CHAPS, $8 \mathrm{M}$ urea and $0.002 \%(\mathrm{w} / \mathrm{v})$ bromophenol blue for $60 \mathrm{~min}$ at $20^{\circ} \mathrm{C}$. This solution was then used to reswell a $24 \mathrm{~cm}$ immobilized $\mathrm{pH}$ gradient strip, pH 4-7 (GE Healthcare), for at least $12 \mathrm{~h}$. After rehydration the strip was electrophoresed at 100, 200, 500 and $2000 \mathrm{~V}$ for $1 \mathrm{~h}$ each, followed by $4000 \mathrm{~V}$ to $\sim 72000 \mathrm{~V} \mathrm{~h}$, and finally $8000 \mathrm{~V}$ for $1 \mathrm{~h}$, with the current set at $50 \mu \mathrm{A}$ per gel for the duration of the run. Strips were either stored at $-70{ }^{\circ} \mathrm{C}$ until required, or equilibrated prior to electrophoresis in the second dimension.

SDS-PAGE. IEF strips were incubated in SDS equilibration buffer [0.05 M Tris, $6 \mathrm{M}$ urea, $30 \%$ (v/v) glycerol, $2 \%(\mathrm{w} / \mathrm{v})$ SDS and $0.002 \%(\mathrm{w} / \mathrm{v})$ bromophenol blue] containing $0.065 \mathrm{M}$ DTT for $15 \mathrm{~min}$ with gentle shaking, followed by similar incubation in a fresh aliquot of equilibrium buffer containing iodoacetamide $(0.135 \mathrm{M})$. Polyacrylamide gels $[26 \times 20 \mathrm{~cm}, 12.5 \%(\mathrm{w} / \mathrm{v})$; GE Healthcare] were electrophoresed using an Ettan Dalt system (GE Healthcare), at $1 \mathrm{~W}$ per gel, overnight at $15^{\circ} \mathrm{C}$. The power was then increased to $2-4 \mathrm{~W}$ per gel until the dye-front was within $1 \mathrm{~cm}$ of the bottom of the gel.

Visualization of proteins. Gels were stained either with silver using the method described by Morrissey (1981), with the exception that the glutaraldehyde fixation (step 2) was omitted, or with colloidal CBB using the method described by Neuhoff et al. (1985).

Scanning and comparison of gels. Scanned gel images were obtained using a Umax PowerLook III Imagescanner coupled with Imagemaster Labscan version 3.01 software (GE Healthcare). Image analyses and gel comparisons were subsequently performed using Progenesis PG200 software (Nonlinear Dynamics). Gels were automatically analysed using the spot detection feature of the software (method 2005), with automated matching (vector box size 12, search box 64, using both automatic and user-generated seeds and warping). Spot volumes were corrected for background using the 'mode of non-spot method', and normalized as a proportion of the total spot volume of the gel.

Identification of proteins. Proteins of interest were excised from gels with either a $15 \mathrm{~mm}$ or $30 \mathrm{~mm}$ 'spot picker' (The Gel Company), and cut into small pieces $\sim 1-2 \mathrm{~mm}$ in diameter. Gel pieces were incubated with $0.1 \mathrm{M}$ ammonium bicarbonate and $50 \%$ $(\mathrm{v} / \mathrm{v})$ acetonitrile $(\mathrm{ACN})$ for $15 \mathrm{~min}$ at room temperature, with at least three changes until the stain had been removed. Finally the gel pieces were dehydrated with $100 \%$ (v/v) ACN for 10 min. The ACN was removed and the gel pieces were dried using a Speed-Vac (ThermoElectron). The gel pieces were rehydrated in $0.01 \mathrm{M}$ DTT and $0.1 \mathrm{M}$ ammonium bicarbonate, and were incubated at $56^{\circ} \mathrm{C}$ for $1 \mathrm{~h}$, followed by treatment with $0.055 \mathrm{M}$ iodoacetamide and $0.1 \mathrm{M}$ ammonium bicarbonate for $30 \mathrm{~min}$ at room temperature in the dark. Gel pieces were washed with $0.1 \mathrm{M}$ ammonium bicarbonate and $50 \%(\mathrm{v} / \mathrm{v}) \mathrm{ACN}$, with at least two changes, dehydrated in $100 \%$ $(\mathrm{v} / \mathrm{v}) \mathrm{ACN}$ for $10 \mathrm{~min}$, and then dried on the Speed-Vac for $20 \mathrm{~min}$. Proteolytic digestion was carried out using $10 \mathrm{ng}$ trypsin $\mu \mathrm{l}^{-1}$ (sequencing grade modified, Promega, in $0.025 \mathrm{M}$ ammonium bicarbonate) at $37^{\circ} \mathrm{C}$ for at least $16 \mathrm{~h}$. Protein digest $(0.5 \mu \mathrm{l})$ was mixed with $0.5 \mu \mathrm{l}$ of a solution containing $10 \mathrm{mg}$ alpha-cyano-4hydroxycinnamic acid $\mathrm{ml}^{-1}, 50 \%(\mathrm{v} / \mathrm{v}) \mathrm{ACN}$ and $0.1 \%(\mathrm{v} / \mathrm{v})$ trifluoroacetic acid, for analysis by MALDI-TOF on a Voyager-DE Pro mass spectrometer (PerSeptive Biosystems) selecting for a mass range of $600-5000 \mathrm{Da}$.

Silver-stained spots were first destained using a commercial kit (SilverQuest; Invitrogen) prior to the first dehydration step. All subsequent steps were identical to those used for CBB-stained spots, as described above.

MS. Data Explorer was used to create the peak list from the raw data, with the smoothing function applied, signal-to-noise correlation factor set at 0.7, and the data baseline-corrected with the following parameters: peak width 32 , flexibility 0.5 and degree 0.1 . 
The peak height at which centroids were calculated was $50 \%$, and peaks were de-isotoped. Resolution for MS was $>10000$ with a mass accuracy of $\pm 0.01 \%$. A close-external means of calibrating each spectrum, and no means of exclusion of known contaminant ions (such as keratin), were employed.

Bioinformatics. The genome of $M$. avium subsp. paratuberculosis strain K10 (GenBank accession nos. NC_002944 and AE016958) contains 4350 predicted ORFs which were used to compile a protein database, and this was queried using Mascot 2.0 (Matrix Science) (Perkins et al., 1999). Mass data that did not give a significant hit against the K10 database were then used to query the National Center For Biotechnology Information (NCBI) nr database. Searches for trypsin cleavage patterns used a fragment ion mass accuracy of 100 p.p.m., carbamidomethyl modification was selected, and up to one missed cleavage site was permitted. Proteins identified using this procedure were characterized using the Entrez nr Peptide Sequence database (NCBI), using the protein-protein BLAST program. The NCBI Conserved Domain Search service was used to identify domains present in protein query sequences, and the Kyoto Encyclopedia of Genes and Genomes (http://www.kegg.com/) was used to identify relevant metabolic pathways.

\section{RESULTS}

\section{In vivo proteome}

The in vivo proteome generated from M. avium subsp. paratuberculosis scraped from the terminal ileum of sheep M64/02 is presented in Fig. 1(a). The 2D electrophoretogram was stained using the modified silver staining protocol (Morrissey, 1981). The proteome generated was a representation of $\sim 1000$ well-resolved protein spots. The parameters of separation gave an even spread of protein across the electrophoretogram, enabling picking of individual spots. The location of spots was sufficiently consistent between gels to allow recognition of patterns and matching of spots between gels. Variation of spot intensity between analytical replicates is inherent in the technical procedure; therefore, analysis of multiple biological and analytical replicates was required. However, the sample of protein obtained from $M$. avium subsp. paratuberculosis scraped from the terminal ileum of a sheep is a finite resource, with $\leqslant 500 \mu$ g protein recovered per animal, sufficient to run one or two $2 \mathrm{D}$ gels at most.

Microscopic examination of the in vivo density-gradientpurified cell pellet revealed that non-acid-fast material, probably of host gut origin, was present $(\sim 1 \%$ contamination). In order to identify and eliminate these proteins from our analyses, samples from sheep with paucibacillary paratuberculosis were prepared for $2 \mathrm{D}$ electrophoresis in the same manner as for the multibacillary cases. The proteome from paucibacillary paratuberculosis was the preferred control, since it may have been expected to contain host proteins associated with the inflammatory response that would have been absent in the normal gut. The paucibacillary paratuberculosis proteome mainly contained proteins of the inflamed gut, with minimal input from $M$. avium subsp. paratuberculosis (as there were too few bacteria present), and is presented in Fig. 1(b). These proteins (of which there are eight major groups) align on the multi- and paucibacillary M. avium subsp. paratuberculosis proteomes, and have tryptic peptide mass profiles with significant hits to mammalian proteins in the NCBI database (including protein disulfide, helicase, catalase, collagen, chaperonin, bovine ATPase and actin).

\section{In vitro proteome}

The in vitro proteome was generated by extraction and separation of $M$. avium subsp. paratuberculosis grown under optimal conditions in the laboratory. Thus, the proteome generated constituted a picture of the proteins expressed during optimal unrestrictive conditions, quite unlike those experienced during infection. As with the in vivo proteome, silver-stained 2D electrophoretograms generated an in vitro proteome of $\sim 1000$ well-resolved protein spots. Submitting in vitro-grown $M$. avium subsp. paratuberculosis to the same procedure used to purify M. avium subsp. paratuberculosis from the intestine of animals with paratuberculosis did not significantly alter this profile (data not shown).

\section{Choice of stains for visualization of the proteome}

Silver staining of electrophoretograms was necessary for visualization of low-abundance proteins, due to its high degree of sensitivity. However, silver staining is not quantitative, so a rigorous statistical comparison of spot intensities from different gels was not possible. Increasing the protein loading of gels by approximately threefold, and staining with colloidal CBB allowed many of the abundant proteins to be visualized; however, the less abundant proteins (which included most of the proteins upregulated in vivo) could not be visualized. Further increases in loading resulted in loss of resolution. Thus, we continued to use silver-stained gels to identify modulated proteins. Differentially expressed proteins demonstrated altered staining between in vivo and in vitro gels, despite neighbouring proteins having similar levels of staining.

\section{Identification of proteins upregulated in vivo}

M. avium subsp. paratuberculosis from four ovine (M21/02, M64/02, M65/02 and M250/04) and one Moufflon case (M62/02) of multibacillary paratuberculosis were analysed by $2 \mathrm{D}$ electrophoresis (eight gels in total were analysed). For in vitro cultures, an aliquot of cells harvested in either exponential or stationary phase was analysed by $2 \mathrm{D}$ electrophoresis (nine gels in total were analysed). Where possible, in vivo proteomes of each isolate were compared with both the in vitro proteomes (exponential and stationary) derived for that particular isolate, to preclude growth-phase-specific differences. Computer-assisted analysis using Progenesis PG200 software was carried out, which allowed analogous small sections of the proteomes to be viewed simultaneously. Breaking down the complex proteome into smaller regions facilitated the manual detection of protein spots of which the expression appeared 

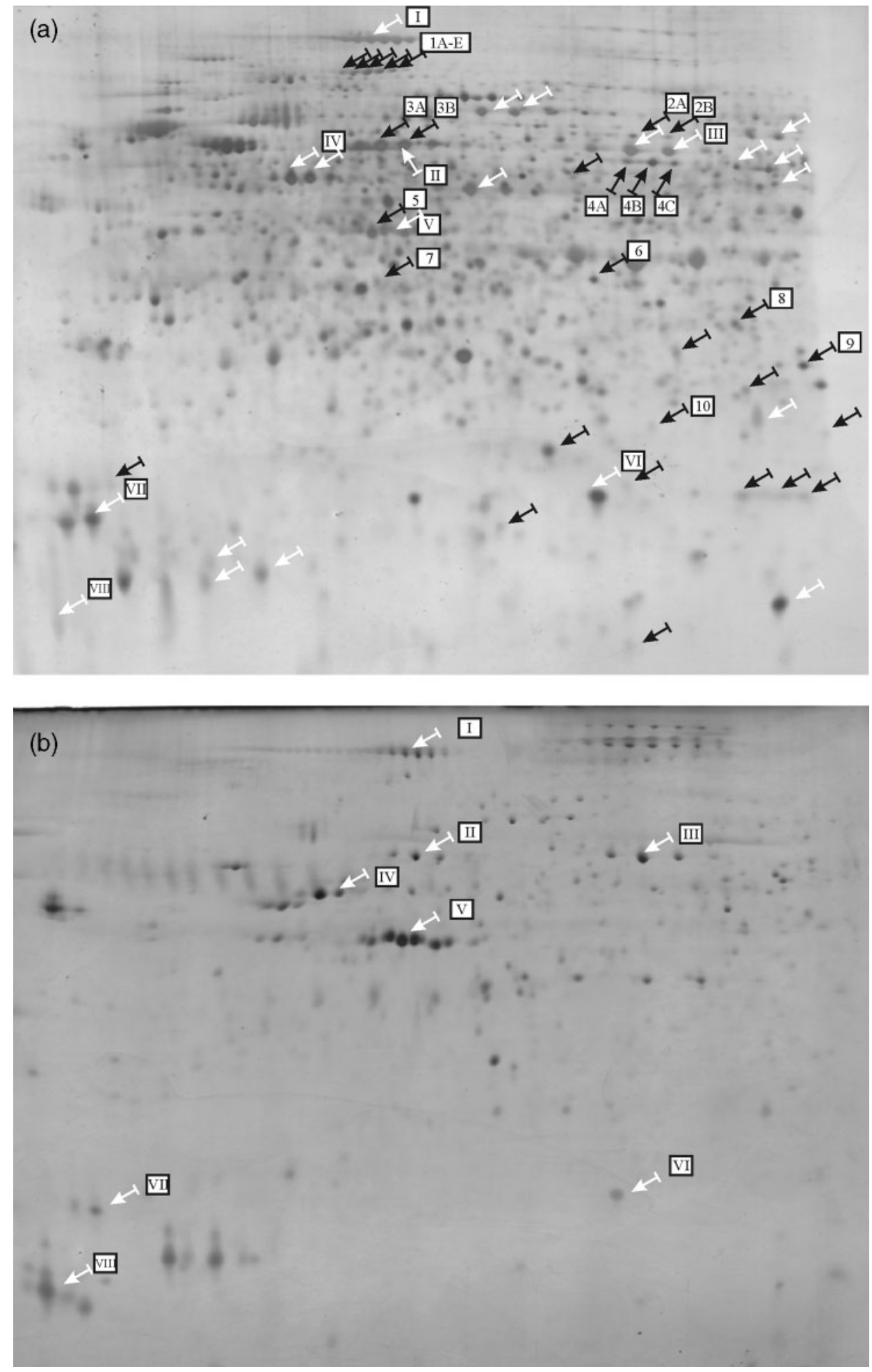

Fig. 1. Silver-stained 2D electrophoretograms of $M$. avium subsp. paratuberculosis scraped from the gut mucosa of cases of clinical multibacillary ovine paratuberculosis (a) and paucibacillary ovine paratuberculosis (b). Proteins which appeared to be upregulated in vivo are highlighted with a black arrow. Spots identified by MALDI-TOF are numbered 1-10, and these correspond to: 1(A-E), ClpB; 2(A, B), RocA; 3(A, B), Fad E14; 4(A-C), MAP 0297c; 5, Arg G; 6, MAP 1754c; 7, SseA; 8, Rsp B; 9, MAP 4227c; 10, PpiA. Ovine gut mucosal proteins not removed by the purification procedure were present in the proteome. These contaminating proteins are indicated by white arrows, and the major contaminants are labelled with Roman numerals to facilitate visualization of the corresponding proteins on the $2 \mathrm{D}$ electrophoretogram of $M$. avium subsp. paratuberculosis from paucibacillary ovine paratuberculosis (b). The figure was compiled using CorelDraw 9 software. 


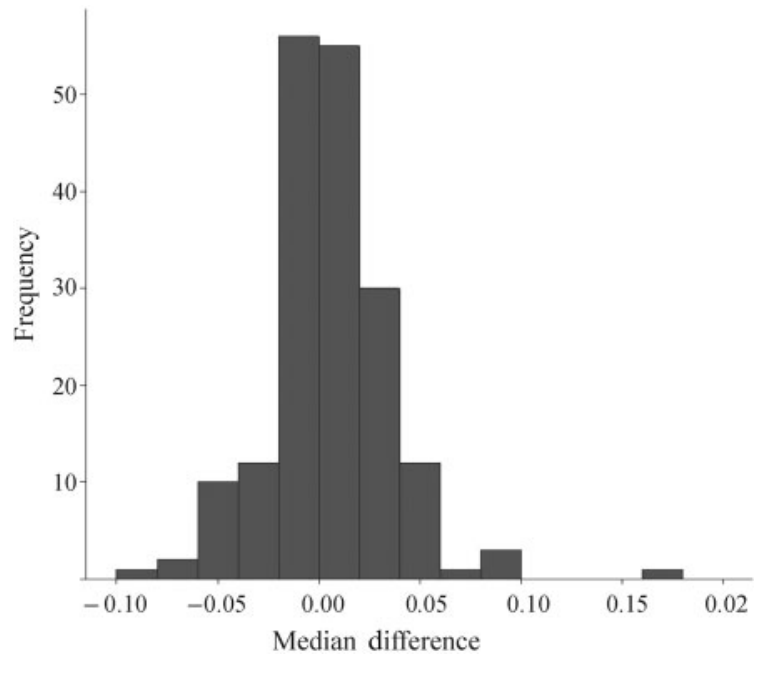

Fig. 2. Distribution of median differences between in vivo and in vitro spot volumes in the selected area (five spots which had much higher volumes than the other spots were not included in this distribution).

to be consistently increased in the proteomes of in vivoisolated organisms. To assess whether the differences in expression observed between the in vivo and in vitro proteomes were likely to be genuine and not the result of experimental variability, an area of the gel with typical spot density was selected for more detailed analysis. This area contained 188 spots, accounting for $\sim 15 \%$ of the spots on the gel (as detected by PG200). The normalized volumes of all spots in the area were recorded for each of the gels (the matches were manually corrected where necessary), and the median volume for each spot was calculated for the in vivo and in vitro gels. The difference in the median value for each spot was then determined. The median value was considered a suitable measure to indicate the typical spot volume, taking into account both biological and technical variability. The difference in the median values, between individual spots on the in vivo and in vitro gels, was used, rather than the percentage increase, because many of the proteins with low expression had a median value of zero. Fig. 2 shows the distribution of median differences between in vivo and in vitro volumes in the selected area. The distribution of differences was fairly symmetrical and centred about zero, which is what would be expected if most proteins behave similarly in vivo and in vitro. Four spots $(2.2 \%$ of the total) had median differences $>0.08$, and these spots appeared to be slightly apart from the rest of the distribution. Therefore, a median difference in spot volume of 0.08 was taken as the cut-off point to indicate an upregulated protein.

Protein spots that appeared to be upregulated in vivo were picked and their tryptic digests analysed by MALDI-TOF. MS data were obtained for proteins, and their tryptic peptide profiles were used to query the $\mathrm{K} 10 \mathrm{M}$. avium subsp. paratuberculosis database using Mascot (Perkins et al., 1999). Ten proteins were identified with a significant hit to the K10 M. avium subsp. paratuberculosis database, and are numbered and indicated by black arrows in Fig. 1(a). Mascot search data for these proteins are presented in Table 1. Identification revealed some spots to be multiple isoelectric forms of the same protein present in horizontal strings (1A-E, $2 \mathrm{~A}$ and $2 \mathrm{~B}$, $3 \mathrm{~A}$ and $3 \mathrm{~B}$ and $4 \mathrm{~A}-\mathrm{C}$ ), probably generated by covalent modifications which altered the pI. Expanded views of selected spots for three strains (M62/02, M64/02 and M65/02) are shown in Fig. 3(a-c). Other upregulated proteins which were not identified are indicated solely by black arrows.

Table 1. Results of queries to the M. avium subsp. paratuberculosis K10 Mascot database for protein spots 1-10

\begin{tabular}{|c|c|c|c|c|c|c|c|c|}
\hline Spot no.* & $\begin{array}{c}\text { MASCOT } \\
\text { score } \dagger\end{array}$ & Annotation & Functionality of protein & $\begin{array}{l}\text { Nominal } \\
\text { mass }(\mathrm{Da})\end{array}$ & $\begin{array}{c}\text { Calculated } \\
\text { pI }\end{array}$ & $\begin{array}{c}\text { Peptide } \\
\text { count }\end{array}$ & $\begin{array}{c}\text { Masses } \\
\text { searched }\end{array}$ & $\begin{array}{c}\text { Sequence } \\
\text { coverage }(\%)\end{array}$ \\
\hline $1 \mathrm{~A}-\mathrm{E}$ & 187 & MAP3853 & ATP-dependent Clp protease $(\mathrm{ClpB})$ & 92575 & 5.19 & 23 & 50 & 31 \\
\hline $3 \mathrm{~A}$ and $\mathrm{B} \ddagger$ & 55 & MAP1553c & $\begin{array}{l}\text { Putative acyl-CoA dehydrogenase } \\
\text { (Fad E14) }\end{array}$ & 41629 & 7.21 & 10 & 110 & 35 \\
\hline 5 & 150 & MAP 1367 & Arginosuccinate synthase (ArgG) & 43912 & 5.21 & 18 & 113 & 70 \\
\hline 6 & 123 & MAP1754c & usp & 31073 & 5.72 & 10 & 24 & 38 \\
\hline 7 & 118 & MAP3402 & Thiosulfate sulfurtransferase (SseA) & 33455 & 5.23 & 12 & 50 & 40 \\
\hline 8 & 104 & MAP $2956 c$ & 30 S ribosomal protein S2 (RpsB) & 30192 & 6.03 & 13 & 102 & 56 \\
\hline 9 & 168 & MAP4227c & $\operatorname{lmo}$ & 30261 & 6.31 & 12 & 26 & 53 \\
\hline
\end{tabular}

${ }^{\star}$ Spot numbers correspond to those in Figs $1($ a) and 3 .

$\dagger$ Threshold value for significance is $52(P<0.05)$.

\$Where more than one spot had the same identity, the best Mascot score was recorded. 
(a)
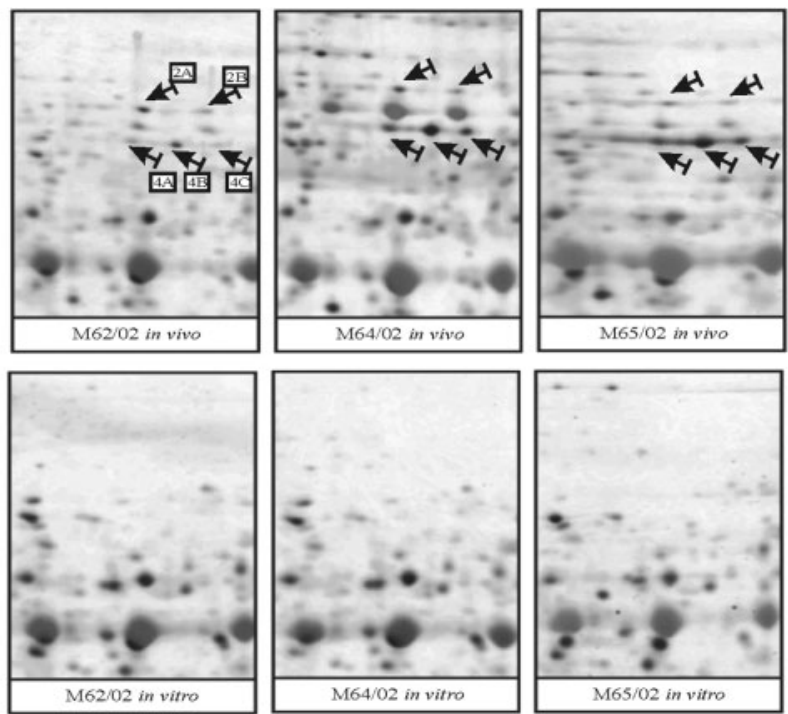

(b)
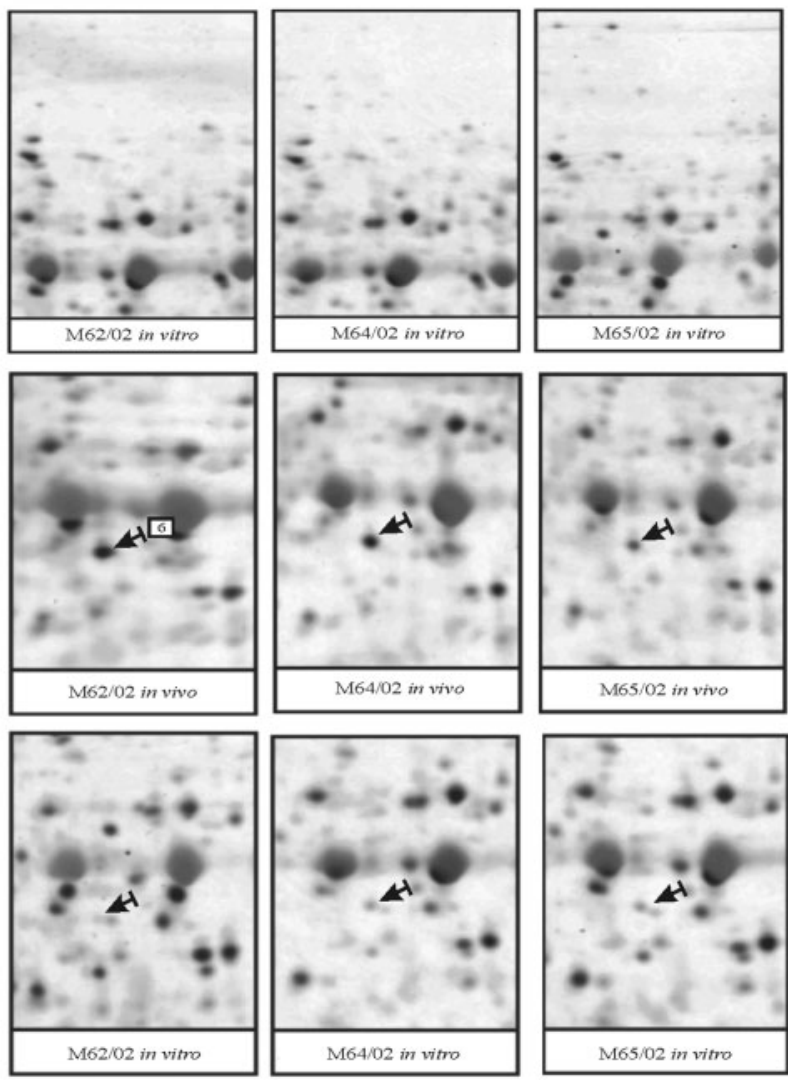

(c)
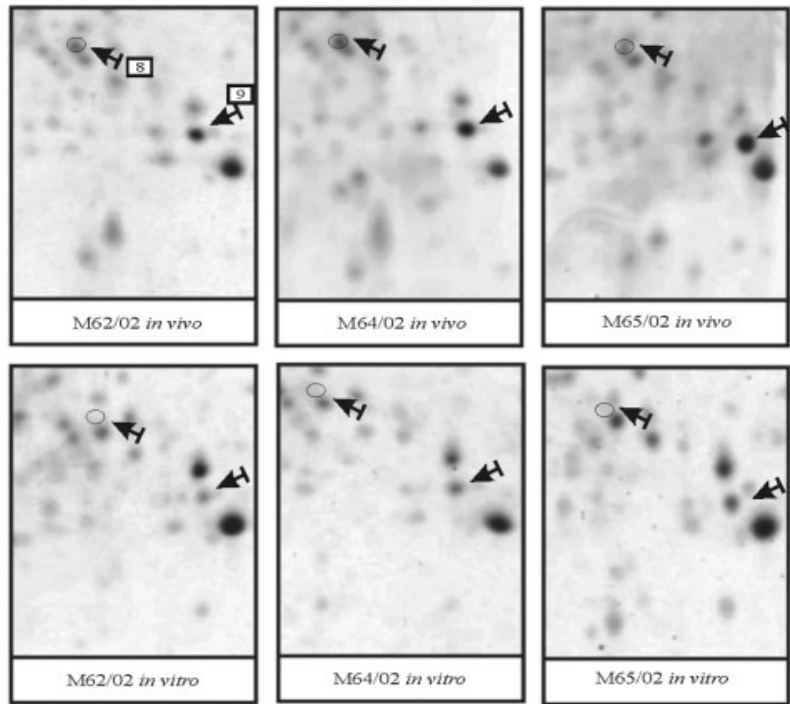

Fig. 3. Expanded regions of $2 D$ electrophoretograms. Upper panels of $(\mathrm{a}-\mathrm{c})$ show expanded regions of silver-stained 2D electrophoretograms of three $M$. avium subsp. paratuberculosis strains scraped from the gut mucosa of three cases of multibacillary paratuberculosis (M62/02, M64/02 and M65/ 02). Lower panels of $(\mathrm{a}-\mathrm{c})$ show analogous regions of silverstained 2D electrophoretograms of the strains isolated from the gut mucosa from the same animal, grown in standard laboratory culture to exponential phase (proteomes of stationary-phase cultures appeared similar to the exponential phase in the areas presented). The numbers correspond to those on the 2D electrophoretogram in Fig. 1(a) and in Tables 1 and 2. The figure was compiled using Phoretix 2D Elite and CorelDraw 9 software.

Table 2 shows the median differences in spot volume of the 10 proteins which were identified. Whilst the criterion (median difference $>0.08$ ) was useful for highlighting upregulated proteins, it was not able to select proteins with small volumes, even if they were consistently expressed in vivo but not in vitro. For example, spots 2 and 7, upon visual examination, appeared more frequently and more intensely in in vivo samples, but the in vivo median values were only 0.04 and 0.05 respectively (in vitro median values were 0 and 0.01 ), and therefore did not meet the criterion.

Upregulated proteins FadE14 (spot 3) and ArgG (spot 5) aligned to spots present in the paucibacillary proteome, and also gave significant hits to mammalian proteins in the NCBI database. To determine whether these proteins were truly upregulated, or only identified due to the presence of co-migrating contaminating protein, we picked multiple spots in the corresponding areas on in vitro gels, to see if these proteins could be detected by MALDI-TOF analysis (even though no visible spot was present on the in vitro gels).
No significant hits were found, and thus it is likely that FadE14 and ArgG represented upregulated proteins of in vivo M. avium subsp. paratuberculosis.

\section{DISCUSSION}

Proteomic analysis of organisms isolated directly from the site of infection is an exploratory approach to study bacterial virulence factors that are critical to successful infection. This is believed to be the first report utilizing this approach to study $M$. avium subsp. paratuberculosis virulence factors. Analysis of M. avium subsp. paratuberculosis isolated from the terminal ileum of ovine paratuberculosis cases identified a set of 10 proteins whose expression is upregulated during natural infection. These proteins were identified as 1pyrroline-5-carboxylate dehydrogenase (RocA), a putative acyl-CoA dehydrogenase (FadE14), 2-methylcitrate dehydratase (2-mcd), arginosuccinate synthase (ArgG), universal stress protein (usp), 30S ribosomal protein S2 (RpsB), 
Table 2. Normalized spot volumes for in vivo upregulated proteins

Where proteins were represented on the proteome as multiple spots they were designated letters in addition to numbers, and in these cases the mean differences are quoted.

\begin{tabular}{|lccc|}
\hline Spot no. & \multicolumn{3}{c|}{ Normalized spot volume } \\
\cline { 2 - 4 } & $\begin{array}{r}\text { Median } \\
\text { in vivo }\end{array}$ & $\begin{array}{l}\text { Median } \\
\text { in vitro }\end{array}$ & $\begin{array}{c}\text { Median } \\
\text { difference }\end{array}$ \\
\hline 1A-E & 0.62 & 0.15 & 0.09 \\
$2 \mathrm{~A}$ and B & 0.08 & 0 & 0.04 \\
$3 \mathrm{~A}$ and B & 0.22 & 0.06 & 0.08 \\
$4 \mathrm{~A}-\mathrm{C}$ & 0.39 & 0.05 & 0.11 \\
5 & 0.14 & 0.03 & 0.11 \\
6 & 0.12 & 0.03 & 0.09 \\
7 & 0.05 & 0.01 & 0.04 \\
8 & 0.21 & 0.11 & 0.10 \\
9 & 0.29 & 0.08 & 0.21 \\
10 & 0.19 & 0.02 & 0.17 \\
\hline
\end{tabular}

peptidyl-prolyl cis-trans isomerase (PpiA), luciferase-like monooxygenase (lmo), thiosulfate sulfurtransferase (SseA) and ATP-dependent Clp protease (ClpB).

It has been proposed that expression of proteins associated with alternative carbon metabolism, iron limitation and cellular hypoxia is necessary for Mycobacterium tuberculosis to survive in granulomatous lesions (Timm et al., 2003). In paratuberculosis, ArgG and RocA may have a role in the adaptation of $M$. avium subsp. paratuberculosis to its niche and the utilization of carbon sources therein. One hypothesis is that $\operatorname{ArgG}$ and RocA have a concerted action for energy production in vivo, shunting carbon units from amino acids to the tricarboxylic acid cycle via glutamate (RocA reduces the accumulation of 1-pyrroline-5-carboxylate, a cytotoxic intermediate of proline or arginine catabolism). These proteins may be important for scavenging arginine and proline from proteins degraded by the host immune response. Alternatively, ArgG may be expressed to synthesize arginine, and although the latter is abundant in dietary sources (it is one of the major fuels in the mucosa; $\mathrm{Wu}, 1998$ ), it may be sequestered and thus limiting for intracellular $M$. avium subsp. paratuberculosis. In addition, because of the high GC content of M. avium subsp. paratuberculosis, its proteins tend to be arginine-rich, so synthesis may need to be increased to provide sufficient arginine for replication and survival of the pathogen. Proline dehydrogenase (encoded by Map 2592c, which resides next to RocA and is potentially coexpressed) may oxidize proline to provide the building blocks, via RocA, for arginine synthesis. The net affect is catabolism of proline and generation of arginine. If arginine synthesis is vitally important for in vivo survival of $M$. avium subsp. paratuberculosis, then this may be a potential intervention point in the control of $M$. avium subsp. paratuberculosis infection. Similarly, in vivo accumulation of 2-mcd involved in propionate metabolism is indicative of
M. avium subsp. paratuberculosis exploiting available substrates; propionate being one of the major volatile fatty acids in the rumen and large intestine. In the list of upregulated proteins, ArgG and RocA are the only examples of proteins functioning in the same pathway. It is probable that other upregulated proteins are indicators of in vivo-modulated pathways, but their components have not been identified due to the limitations of proteomic analysis.

FadE14 (MAP 1533c) overexpression is indicative of a response to restrictive iron concentrations experienced in vivo. In M. tuberculosis, FadE14 is part of an iron and IdeR repressible operon which produces a polycistronic message encoding FadE14, FadD33 and an acyl carrier protein (acp) (Rodriguez et al., 2002). In Mycobacterium smegmatis, FadE14, FadD33 and acp are necessary for wild-type mycobactin production (LaMarca et al., 2004), and are presumed to be involved in the synthesis of its aliphatic sidechains. This structural organization of the gene cluster is preserved in $M$. avium subsp. paratuberculosis. This, together with expression of FadE14, suggests that $M$. avium subsp. paratuberculosis may synthesize mycobactin when present in the gut mucosa; an interesting observation, since $M$. avium subsp. paratuberculosis does not express FadE14, and requires the addition of exogenous mycobactin for growth in vitro.

The finding that MAP 1754c, a homologue of the usp, was upregulated underscores the suggestion that $M$. avium subsp. paratuberculosis responded to the adverse conditions in the pathogenic lesion. Protective mechanisms of the usp family may be due to their action as gene regulators (Nystrom \& Neidhardt, 1996), or involve their direct binding to DNA or interaction with other proteins, possibly modulated by their ability to bind ATP (O'Toole \& Williams, 2003). Rv2005c (the closest M. tuberculosis homologue of MAP 1754c) is upregulated in response to hypoxia, and also in an $h s p R$-deleted mutant (Stewart et al., 2002). In Mycobacterium bovis BCG, usp Rv263 (Boon \& Dick, 2002) is under the control of the two component response regulator DevR (O'Toole et al., 2003), its role in control of usps in M. avium subsp. paratuberculosis remains to be delineated. It was interesting to note the proximal location of the gene containing sigma 70 regions 2 and 4 (MAP 1757c), because in Eschericia coli, uspA is controlled by a single sigma 70 -dependent promoter (Kvint et al., 2003).

The links between in vivo phenotype and the heat-shock response are reinforced by the upregulation of PPiA, ClpB and RpsB. PpiA catalyses the interconversion of cis-trans peptide bonds and can accelerate protein folding, with implications in cell surface recognition. In M. tuberculosis, the protein is upregulated in an $h s p R$ and $h r c A$ doubledeletion mutant; therefore, this protein may be related to the heat-shock response, and possibly act as a virulence factor (Stewart et al., 2002). ClpB is required for intracellular multiplication in bovine epithelial cells and thermotolerance in Staphylococcus aureus (Frees et al., 2004). In E. coli, 
ClpB expression is induced by heat shock (Kim et al., 1998; Squires et al., 1991). ClpB has an indispensable role in the solubilization of larger protein aggregates in cooperation with the DnaK chaperone system (Goloubinoff et al., 1999; Mogk et al., 1999). The finding that RpsB was upregulated is consistent with a general trend for nearly all ribosomal genes to be upregulated in the $h s p R$-deleted mutant (Stewart et al., 2002).

For many of the proteins listed, it was possible to assign their function due to the existence of well-characterized homologues with demonstrated activities. However, this was not the case for proteins SseA (a rhodanese-related sulfurtransferase) and lmo; therefore, their roles in the virulent state of $M$. avium subsp. paratuberculosis are unknown. SSeA of $E$. coli functions as a 3-mercaptopyruvate:cyanide sulfurtransferase in vitro, although its physiological function remains to be elucidated (Colnaghi et al., 2001). The lmo, which also has the related flavin-dependent oxidoreductase motif, has sequence identity to hypothetical proteins only. However, it is notable that they are highly conserved in $M$. tuberculosis and M. bovis, suggesting an important as-yetundiscovered function.

The upregulated proteins indicate that the in vivo state has links to both the heat-shock and hypoxia responses of mycobacteria in vitro. M. avium subsp. paratuberculosis has adapted its proteomic repertoire to exploit the available nutrients and increase its capacity for iron acquisition, and provides an insight into the conditions of the pathogenic lesion. Upregulation of usp MAP $1754 \mathrm{c}$ may be indicative of M. avium subsp. paratuberculosis resistance to host defences. The increased role of PpiA in the pathogenic process, and its potential to alter cell surface recognition, allows speculation that this protein may be involved in stealth, evading the immune response. A recent study of Mycobacterium avium subspecies avium proteins overexpressed in human macrophages has identified proteins FadE2, FixA, KasA and EF-tu (Brunori et al., 2004). It is interesting that there is no overlap in the sets of identified proteins. This may be because only a proportion of upregulated proteins have been detected by both methods. It may also reflect the differences in response of the organisms to the different environments in the studies. The presence of KasA in the list is intriguing, as this was identified as one of the down-regulated proteins in vivo (data not presented). It is interesting to note that none of the proteins regulated in vivo detected by this study is present in the pathogenicity island reported by Stratmann et al. (2004).

The hydrophobic nature of the cell wall (Brennan \& Nikaido, 1995) makes M. avium subsp. paratuberculosis cells prone to aggregation, forming large conglomerates in liquid culture. In this study, stirred cultures (Hughes et al., 2001) produced physiologically homogeneous cells which were harvested in a defined growth phase. This had a significant impact on the quality of the proteomic profile generated, making comparisons easier. Electrophoretograms generated by our procedure compared well with those generated by the recently published method of Lanigan et al. (2004) for 2D gel electrophoresis of M. avium subsp. paratuberculosis and $M$. avium subsp. avium.

M. avium subsp. paratuberculosis from naturally infected animals is a limited resource, and as a result, it has not been possible to obtain sequence data for some low-abundance proteins; indeed, a portion of them will not have been detectable at all. Proteomic analysis of organisms from the paucibacillary form of paratuberculosis has not been possible. More sensitive transcript detection methods, such as selective capture of transcripts analysis (Dozois et al., 2003) or microarray technology, may reveal a greater number of modulated proteins. However, it has recently been reported that the correlation of protein and mRNA expression is poor (Brunori et al., 2004), therefore, protein profiles may reflect more accurately the composition of in vivo $M$. avium subsp. paratuberculosis. It would be advantageous to quantify the transcripts of the identified proteins using real-time PCR, to determine whether the observed increase in expression is due to increased transcription.

M. avium subsp. paratuberculosis scraped from the intestinal mucosa may contain several subpopulations, e.g. intracellular and extracellular organisms, the proportion of which may change throughout the course of infection. Transiently expressed proteins, e.g. those responsible for promoting early infection, may not be detected. Secreted proteins may also not be detected due to the extraction procedures applied. In vitro studies and experimental infections, although difficult to establish in the natural host, may address this. The temporal and tissue-specific expression of proteins that facilitate entry, replication and persistence of $M$. avium subsp. paratuberculosis during the progression of paratuberculosis will provide insight into host-pathogen interactions.

Inevitably, with global approaches a list of proteins of interest is generated, and the challenge is to understand how these subtle changes in proteome, brought about through interaction with the host, can promote bacterial growth in vivo. In this study, we identified a number of core genes involved in metabolism and adaptation to physiological change, expressed in the host environment. If a pathogen increases its expression of a set of genes during growth within the host, it is likely that these genes will play a role in virulence. We are now in a position to test this hypothesis in M. avium subsp. paratuberculosis.

\section{ACKNOWLEDGEMENTS}

The work was funded by the Scottish Executive Environment and Rural Affairs Department (SEERAD) and the European Union (contract number QLK2-CT-2001-01420).

We would like to thank staff at the Moredun Research Institute: Linda May and Susan Denham for their expertise in bacteriological culture; the Functional Genomic Unit for MALDI-TOF analysis; Dr Craig Watkins and Michael Malcom-Smith for assistance at post-mortems; Dr Alex Lainson and Raja Yaga for preparing the Mascot database; and 
the Clinical Department for Animal Husbandry. We are grateful to Dr Susan Rhind (Easter Bush Veterinary Centre, University of Edinburgh) for pathology. Finally, but not least, we thank local farmers for providing clinical cases of paratuberculosis for this study.

\section{REFERENCES}

Angelichio, M. J. \& Camilli, A. (2002). In vivo expression technology. Infect Immun 70, 6518-6523.

Boon, C. \& Dick, T. (2002). Mycobacterium bovis BCG response regulator essential for hypoxic dormancy. J Bacteriol 184, 6760-6767.

Brennan, P. J. \& Nikaido, H. (1995). The envelope of mycobacteria. Annu Rev Biochem 64, 29-63.

Brunori, L., Giannoni, F., Bini, L., Liberatori, S., Frota, C., Jenner, P., Thoresen, O. F., Orefici, G. \& Fattorini, L. (2004). Induction of Mycobacterium avium proteins upon infection of human macrophages. Proteomics 4, 3078-3083.

Chiodini, R. J., van Kruiningen, H. J. \& Merkal, R. S. (1984). Ruminant paratuberculosis (Johnes disease): the current status and future prospects. Cornell Vet 74, 218-262.

Choy, E., Whittington, R. J., Marsh, I., Marshall, J. \& Campbell, M. T. (1998). A method for purification and characterisation of Mycobacterium avium subsp. paratuberculosis from the intestinal mucosa of sheep with Johnes disease. Vet Microbiol 64, 51-60.

Clarke, C. J. (1997). The pathology and pathogenesis of paratuberculosis in ruminants and other species. J Comp Pathol 116, 217-261.

Colnaghi, R., Cassinelli, G., Drummond, M., Forlani, F. \& Pagani, S. (2001). Properties of the Escherichia coli rhodanese-like protein SseA: contribution of the active-site residue Ser240 to sulfur donor recognition. FEBS Lett 500, 153-156.

Dozois, C. M., Daigle, F. \& Curtiss, R. (2003). Identification of pathogen-specific and conserved genes expressed in vivo by an avian pathogenic Escherichia coli strain. Proc Natl Acad Sci U S A 100, 247-252.

Frees, D., Chastanet, A., Qazi, S., Sorensen, K., Hill, P., Msadek, T. \& Ingmer, H. (2004). Clp ATPases are required for stress tolerance, intracellular replication and biofilm formation in Staphylococcus aureus. Mol Microbiol 54, 1445-1462.

Goloubinoff, P., Mogk, A., Peres Ben Zvi, A., Tomoyasu, T. \& Bukau, B. (1999). Sequential mechanism of solubilization and refolding of stable protein aggregates by a bichaperone network. Proc Natl Acad Sci U S A 96, 13732-13737.

Hughes, V. M., Stevenson, K. \& Sharp, J. M. (2001). Improved preparation of high molecular weight DNA for pulsed-field gel electrophoresis from mycobacteria. J Microbiol Methods 44, 209-215. Julian, R. J. (1975). A short review and some observations on Johne's disease with recommendations for control. Can Vet J 16, 33-43.

Kim, K. I., Woo, K. M., Seong, I. S., Lee, Z. W., Baek, S. H. \& Chung, C. H. (1998). Mutational analysis of the two ATP-binding sites in $\mathrm{ClpB}$, a heat shock protein with protein activated ATPase activity in Eschericia coli. Biochem J 333, 671-676.

Kvint, K., Nachin, L., Diez, A. \& Nystrom, T. (2003). The bacterial universal stress protein: function and regulation. Curr Opin Microbiol 6, 140-145.
LaMarca, B. B. D., Zhu, W., Arceneaux, J. E. L., Byers, B. R. \& Lundrigan, M. D. (2004). Participation of $\mathrm{fad}$ and $\mathrm{mbt}$ genes in synthesis of mycobactin in Mycobacterium smegmatis. J Bacteriol 186, 374-382.

Lanigan, M. D., Vaughan, J. A., Shiell, B. J., Beddome, G. J. \& Michalski, W. P. (2004). Mycobacterial proteome extraction: comparison of disruption methods. Proteomics 4, 1094-1100.

Mogk, A., Tomoyasu, T., Goloubinoff, P., Rudiger, S., Roder, D., Langen, H. \& Bukau, B. (1999). Identification of thermolabile Escherichia coli proteins: prevention and reversion of aggregation by DnaK and ClpB. EMBO J 18, 6943-6949.

Morrissey, J. H. (1981). Silver stain for proteins in polyacrylamide gels-a modified procedure with enhanced uniform sensitivity. Anal Biochem 117, 307-310.

Neuhoff, V., Stamm, R. \& Eibl, H. (1985). Clear background and highly sensitive protein staining with coomassie blue dyes in polyacrylamide gels-a systematic analysis. Electrophoresis 6, 427-448.

Nystrom, T. \& Neidhardt, F. C. (1996). Effects of overproducing the universal stress protein, UspA, in Escherichia coli K-12. J Bacteriol 178, 927-930.

O'Toole, R. \& Williams, H. D. (2003). Universal stress proteins and Mycobacterium tuberculosis. Res Microbiol 154, 387-392.

O'Toole, R., Smeulders, M. J., Blokpoel, M. C., Kay, E. J., Loughheed, K. \& Williams, H. D. (2003). A two-component regulator of universal stress protein expression and adaptation to oxygen starvation in Mycobacterium smegmatis. J Bacteriol 185, 1543-1554.

Perkins, D. N., Pappin, D. J., Creasy, D. M. \& Cottrell, J. S. (1999). Probability-based protein identification by searching sequence databases using mass spectrometry data. Electrophoresis 20, 3551-3567.

Rodriguez, G. M., Voskuil, M. I., Gold, B., Schoolnik, G. K. \& Smith, I. (2002). IdeR, An essential gene in Mycobacterium tuberculosis: role of IdeR in iron-dependent gene expression, iron metabolism, and oxidative stress response. Infect Immun 70, 3371-3381.

Squires, C. L., Pedersen, S. B., Ross, M. \& Squires, C. (1991). $\mathrm{ClpB}$ is the Escherichia coli heat shock protein F84.1. J Bacteriol 173, 4254-4262.

Stewart, G. R., Wernisch, L., Stabler, R., Mangan, J. A., Hinds, J., Laing, K. G., Young, D. B. \& Butcher, P. D. (2002). Dissection of the heat-shock response in Mycobacterium tuberculosis using mutants and microarrays. Microbiology 148, 3129-3137.

Timm, J., Post, F. A., Bekker, L.-G., Walther, G. B., Wainwright, H. C., Manganelli, R., Chan, W. T., Tsenova, L., Gold, B. \& other authors (2003). Differential expression of iron-, carbon-, and oxygen-responsive mycobacterial genes in the lungs of chronically infected mice and tuberculosis patients. Proc Natl Acad Sci U S A 100, 14321-14326.

Stratmann, J., Strommenger, B., Goethe, R., Dohmann, K., Gerlach, G. F., Stevenson, K., Li, L. L., Zhang, Q., Kapur, V. \& Bull, T. J. (2004). A 38-kilobase pathogenicity island specific for Mycobacterium avium subsp. paratuberculosis encodes cell surface proteins expressed in the host. Infect Immun 72, 1265-1274.

Wu, G. (1998). Intestinal mucosal amino acid catabolism. J Nutr 128, 1249-1252.

Edited by: W. Bitter 